\title{
BMJ Open Impact of infectious diseases consultation on the management of Staphylococcus aureus bacteraemia in children
}

\author{
Rebecca B Saunderson, ${ }^{1}$ Theodore Gouliouris, ${ }^{1,2,3}$ Edward J Cartwright, ${ }^{1,3}$ \\ Emma J Nickerson, ${ }^{4}$ Sani H Aliyu, ${ }^{2,4}$ D Roddy O'Donnell, ${ }^{5}$ Wilf Kelsall, ${ }^{5}$ \\ D Limmathurotsakul, ${ }^{6}$ Sharon J Peacock, ${ }^{1,2,3,7}$ M Estée Török ${ }^{1,2,3}$
}

To cite: Saunderson RB, Gouliouris T, Cartwright EJ, et al. Impact of infectious diseases consultation on the management of Staphylococcus aureus bacteraemia in children. BMJ Open 2014;4:e004659. doi:10.1136/bmjopen-2013004659

- Prepublication history for this paper is available online. To view these files please visit the journal online (http://dx.doi.org/10.1136/ bmjopen-2013-004659).

Received 10 December 2013 Revised 2 June 2014 Accepted 12 June 2014

CrossMark

For numbered affiliations see end of article.

Correspondence to Dr M Estée Török; et317@medschl.cam.ac.uk

\section{ABSTRACT}

Objectives: Infectious diseases consultation (IDC) in adults with Staphylococcus aureus bacteraemia (SAB) has been shown to improve management and outcome. The aim of this study was to evaluate the impact of IDC on the management of SAB in children.

Study design: Observational cohort study of children with SAB.

Setting: Cambridge University Hospitals National Health Service (NHS) Foundation Trust, a large acute NHS Trust in the UK.

Participants: All children with SAB admitted to the Cambridge University Hospitals NHS Foundation Trust between 16 July 2006 and 31 December 2012.

Methods: Children with SAB between 2006 and 31 October 2009 were managed by routine clinical care (pre-IDC group) and data were collected retrospectively by case notes review. An IDC service for SAB was introduced in November 2009. All children with $S A B$ were reviewed regularly and data were collected prospectively (IDC group) until 31 December 2012. Baseline characteristics, quality metrics and outcome were compared between the pre-IDC group and IDC group.

Results: There were 66 episodes of $S A B$ in 63 children-28 patients (30 episodes) in the pre-IDC group, and 35 patients ( 36 episodes) in the IDC group. The median age was 3.4 years (IQR $0.2-10.7$ years). Patients in the IDC group were more likely to have echocardiography performed, a removable focus of infection identified and to receive a longer course of intravenous antimicrobial therapy. There were no differences in total duration of antibiotic therapy, duration of hospital admission or outcome at 30 or 90 days following onset of $S A B$.

Conclusions: IDC resulted in improvements in the investigation and management of $S A B$ in children.

\section{INTRODUCTION}

Staphylococcus aureus bacteraemia (SAB) is a serious infection that leads to significant morbidity and mortality in adults and children. ${ }^{1}{ }^{2}$

\section{Strengths and limitations of this study}

- This is the first study to examine the impact of introduction of infectious diseases consultation (IDC) on the management of Staphylococcus aureus bacteraemia (SAB) in children.

- We found that IDC was associated with an improvement in investigation and management of $S A B$, but there was no difference in mortality between the pre-IDC and IDC groups.

- The main limitation of the study was the size of the study population, which may explain the lack of mortality benefit.

- The study was conducted in a tertiary referral centre, where clinical management is likely to have been good prior to introduction of the IDC, and may not be generalisable to other settings.

S. aureus causes significant disease in the paediatric population, occurring in $1.5 \%$ of all neonatal intensive care unit (ICU) admissions, ${ }^{3}$ and 6/100 000 children older than 1 year of age. ${ }^{4}$ In neonates, SAB is almost always hospital acquired, and is frequently due to intravascular catheter (IVC)-associated infections. ${ }^{3-6}$ The majority of non-neonatal cases of SAB are community-acquired; those that are hospital-acquired infections are usually IVC associated. ${ }^{7}$

Identified risk factors for the development of $\mathrm{SAB}$ in the paediatric population include having a pre-existing medical condition, prolonged hospitalisation, the presence of an IVC and HIV infection. ${ }^{1} \quad 3-6 \quad 9 \quad 10$ Mortality from $\mathrm{SAB}$ in the adult population is about $30 \% .{ }^{11}$ Mortality rates in the paediatric population tend to be lower, but can be up to $15 \%$ in neonates and/or children with comorbidities. ${ }^{1} 5{ }^{5} \quad 12 \quad 13$ Given that SAB causes a substantial burden of disease in the paediatric population, strategies to improve 
management, prevent the complications of $\mathrm{SAB}$ and reduce mortality are a clinical priority.

The impact of infectious disease consultation (IDC) in adults with $\mathrm{SAB}$ has been extensively studied. ${ }^{14-23}$ IDC has been associated with improved adherence to guidelines, including appropriate and targeted investigation, optimal duration of antibiotic therapy and a reduction in complicated infection, morbidity and mortality. ${ }^{14}$ 16-18 22 24-27 In contrast, the impact of an IDC on the management and outcomes of SAB in children has not previously been evaluated. The aim of this study was to determine the effects of routine IDC on the investigation, management and outcome of children with SAB.

\section{MATERIALS AND METHODS}

\section{Study setting and participants}

Cambridge University Hospitals National Health Service Foundation Trust (CUH) is a tertiary referral centre for paediatrics in the east of England. The paediatric service has a 22-bed medical and surgical ward, a 17-bed paediatric haematology and oncology ward, an 11-bed paediatric ICU and high dependency unit (caring for children aged from 0 to 16 years) and a 12-bed surgical and medical ward for children aged up to 3 years. The Rosie Hospital, the on-site mother and baby hospital, has a 17-cot neonatal ICU and a 10-cot Special Care Baby Unit.

\section{Study design}

We conducted an observational cohort study of all children with SAB admitted to CUH between 16 July 2006 and 31 December 2012. In November 2009, an IDC service for all patients with $\mathrm{SAB}$ was established at CUH. Data were collected from 2006 to 2009 by a retrospective review of the medical records, and prospectively thereafter during the IDC service. Patients with blood cultures that were considered to be contaminants (afebrile with no clinical evidence of infection) or with polymicrobial blood cultures were excluded from the analysis.

\section{Microbiological investigation}

Blood cultures were collected and incubated at $37^{\circ} \mathrm{C}$ for 5 days using the BacT/Alert 3D system (bioMérieux, Basingstoke, UK). Blood cultures that flagged positive were examined by microscopy and presumptively identified as $S$. aureus using a thermostable nuclease test. ${ }^{28}$ Colonies of $S$. aureus were identified by routine methods after a further overnight incubation. Identification of $S$. aureus was performed using a commercial latex agglutination test (Staphaurex, Oxoid Ltd, Basingstoke, UK) until 2011 and then using matrix-assisted laser desorption ionisation time-of-flight mass spectroscopy (Bruker Daltonik, Bremen, Germany). Antibiotic susceptibilities were determined using disc diffusion testing, according to the British Society for Antimicrobial Chemotherapy standards. ${ }^{29}$ Throughout the study period a clinical microbiologist provided telephone advice to the clinical team for all patients with SAB, and attended weekly ward rounds on the paediatric oncology ward and paediatric ICU.

\section{Study procedures}

Prior to November 2009, all patients with $S$. aureus bacteraemia were managed by their primary clinical care team, with telephone advice from the microbiologists. From November 2009, all patients with SAB were reviewed by an infectious disease Specialist Registrar or Consultant, following presumptive identification of $S$. aureus in blood cultures. The assessment included clinical history to determine symptoms of infection, and physical examination to determine possible foci of infection. Patients underwent clinical review daily by their primary care team and at least weekly by the IDC team during their inpatient stay. Demographic, clinical and microbiology data were collected using a standard case record form, and entered into an electronic database.

An IVC was considered to be the focus of infection if there was evidence of inflammation at the catheter exit site and/or a vascular catheter tip culture positive for $S$. aureus, without clinical evidence of another source of bacteraemia. ${ }^{30}$ Thrombophlebitis was diagnosed when there was clinical evidence of infection and inflammation along a blood vessel or when ultrasound or other imaging confirmed the presence of intravascular thrombosis in the setting of suspected infection. Bone and joint infections were defined according to the USA Centers for Disease Control and Prevention criteria. ${ }^{31}$ The lung was considered to be the source of infection when there was clinical, radiological and/or microbiological evidence of pulmonary infection. Soft tissue infection was considered to be the source of the bacteraemia if the clinical signs of a known or suspected soft tissue infection predated or were present at the time of bacteraemia. ${ }^{14}$ A deep tissue abscess was defined by radiological imaging criteria. Infective endocarditis (IE) was diagnosed according to the modified Duke criteria. 3233

A SAB episode was defined as being greater than or equal to 14 days from a previous episode, in the absence of persistent bacteraemia or focus of infection. A secondary site of infection was defined as a site of infection separate from the primary site of infection that was not present at the time of the initial examination. Healthcare-associated bloodstream infection was defined according to previously published criteria. ${ }^{34}$ Hospitalacquired infection was defined according to the USA Centers for Disease Control and Prevention. ${ }^{31}$ Community-acquired infections were defined as those patients with a positive blood culture taken at or within $48 \mathrm{~h}$ of admission who did not meet criteria for healthcare-associated bloodstream infection. ${ }^{34}$ Patients were classified as having uncomplicated $\mathrm{SAB}$ if blood cultures were negative 2-4 days after the initial blood culture was positive, if they had defervesced at $72 \mathrm{~h}$, if 
there was no evidence of metastatic disease or endocarditis or if they had a catheter-related infection. ${ }^{35}$

Appropriate antimicrobial therapy was defined as therapy to which the isolate was determined to be susceptible by antimicrobial disc susceptibility testing. The duration of therapy was the length of time that a patient received antibiotics to which the isolate was susceptible. An underlying medical condition was defined as any chronic medical condition that was present at the time of bacteraemia. Serum C reactive protein, blood white cell counts and platelet counts were measured on the day of, or within $48 \mathrm{~h}$ postbacteraemia. Duration of hospital admission and outcome at 30 and 90 days postbacteraemia was recorded for all patients.

\section{Treatment recommendations}

Antimicrobial treatment recommendations were provided for all children with $\mathrm{SAB}$, based on existing evidence on the management of SAB in adults. ${ }^{14} 36-39$ These included removal of a removable focus of infection, ${ }^{14}$ performing repeat blood cultures at $48-96 \mathrm{~h},{ }^{36}$ performing a transthoracic echocardiogram, performing radiological imaging of suspected deep foci of infection, treating uncomplicated infection with 14 days of intravenous antibiotics, ${ }^{37}$ treating complicated infections with a minimum of 28 days of intravenous antibiotics, ${ }^{38}$ and using $\beta$-lactam therapy as the mainstay of treatment for methicillin-susceptible $S$. aureus. $^{39}$

\section{STATISTICAL ANALYSIS}

Data were analysed using STATA V.12 (StataCorp, College Station, Texas, USA). Categorical variables were analysed using Fisher's exact test and reported as the number and per cent. Continuous variables were compared using the Mann Whitney $\mathrm{U}$ test and reported as the median and IQR. Mortality was analysed per patient (ie, only the first bacteraemia episode was analysed).

\section{ETHICS STATEMENT}

Written informed consent from participants was not required as the study was conducted as a service evaluation.

\section{RESULTS}

\section{Patient characteristics}

Between July 2006 and December 2012, 71 children had one or more blood cultures that were positive for $S$. aureus. Sixty-three children (66 episodes) were included in the study. Five children (six episodes) were excluded because of polymicrobial bacteraemia and three patients (three episodes) were excluded because the cultures were considered to be contaminants. Thus, 28 patients (30 episodes) were included in the pre-IDC group, and 35 patients (36 episodes) in the IDC group. The study schema is summarised in figure 1 . Four of 30 episodes $(13.3 \%)$ received an IDC before the service was implemented in 2009, and
34 of 36 episodes (94.4\%) received an IDC after the service was implemented in 2009.

The baseline characteristics of the two groups were similar (table 1). The clinical features for SAB were likewise similar, apart from an increased proportion of IVC-related infections in the IDC group $(61.3 \%$ vs $26.7 \%, \mathrm{p}<0.01$; table 2). A higher proportion of patients had an unidentified focus of infection in the pre-IDC group compared with the IDC group $(23.3 \%$ vs $5.6 \%$, $\mathrm{p}=0.07$ ). Risk factors for $\mathrm{SAB}$ were also similar apart from an increased frequency of prosthetic material in the IDC group ( $72.2 \%$ vs $46.7 \%$, $\mathrm{p}=0.04$; table 3$)$.

\section{Clinical management}

A service evaluation of the IDC service was conducted, the results of which are summarised in table 4 . In the IDC group, 34/36 episodes had an infectious diseases review, with a median time to review of 2 days (range 14 days). Patients in the IDC group were more likely to have transthoracic echocardiography performed $(80.6 \%$ vs $33.3 \%, \mathrm{p}<0.01)$. They were also more likely to have a removable focus of infection identified $(43.9 \%$ vs $23.3 \%$, $\mathrm{p}<0.01$ ), although there was no difference between the two groups in the likelihood of removal, or the time to removal. In the IDC group, two patients did not have their IVC removed, despite the recommendation to do so, because of concerns about difficulty in re-establishing vascular access. There was no difference in the number of repeat blood cultures performed between groups.

\section{Antimicrobial therapy}

There was no difference between the two groups in the time taken to initiate appropriate antimicrobial therapy. Patients in the IDC group were more likely to receive a longer duration of intravenous antimicrobial therapy (18 vs 13.5 days, $\mathrm{p}=0.04$ ), although there was no difference in total duration of therapy (intravenous and oral) between the two groups. In patients with complicated $\mathrm{SAB}$, the duration of intravenous antibiotic therapy was longer in the IDC group (22 vs 14 days, $\mathrm{p}=0.02$ ), although there was no difference in total duration of antibiotic therapy (intravenous and oral) between the two groups. Patients in the IDC group were more likely to receive a longer duration of intravenous therapy if their repeat blood culture result was positive $(p<0.01)$. In patients with uncomplicated $\mathrm{SAB}$. there was no difference between groups in the duration of intravenous antibiotics, or the total duration of antibiotic therapy. In terms of compliance with recommended standards for duration of therapy, patients in the IDC group were more likely to meet these standards compared with patients in the non-IDC group, both for complicated $\mathrm{SAB}(42.1 \%$ vs $13.3 \%, \mathrm{p}=0.13)$ and uncomplicated $\mathrm{SAB}$ (68.4\% vs $46.7 \%, \mathrm{p}=0.14)$. There was no difference in the proportion of patients receiving $\beta$-lactam therapy for methicillin-sensitive $S$. aureus bacteraemia between the two groups. 
Figure 1 Study schema of paediatric patients with Staphylococcus aureus bacteraemia.

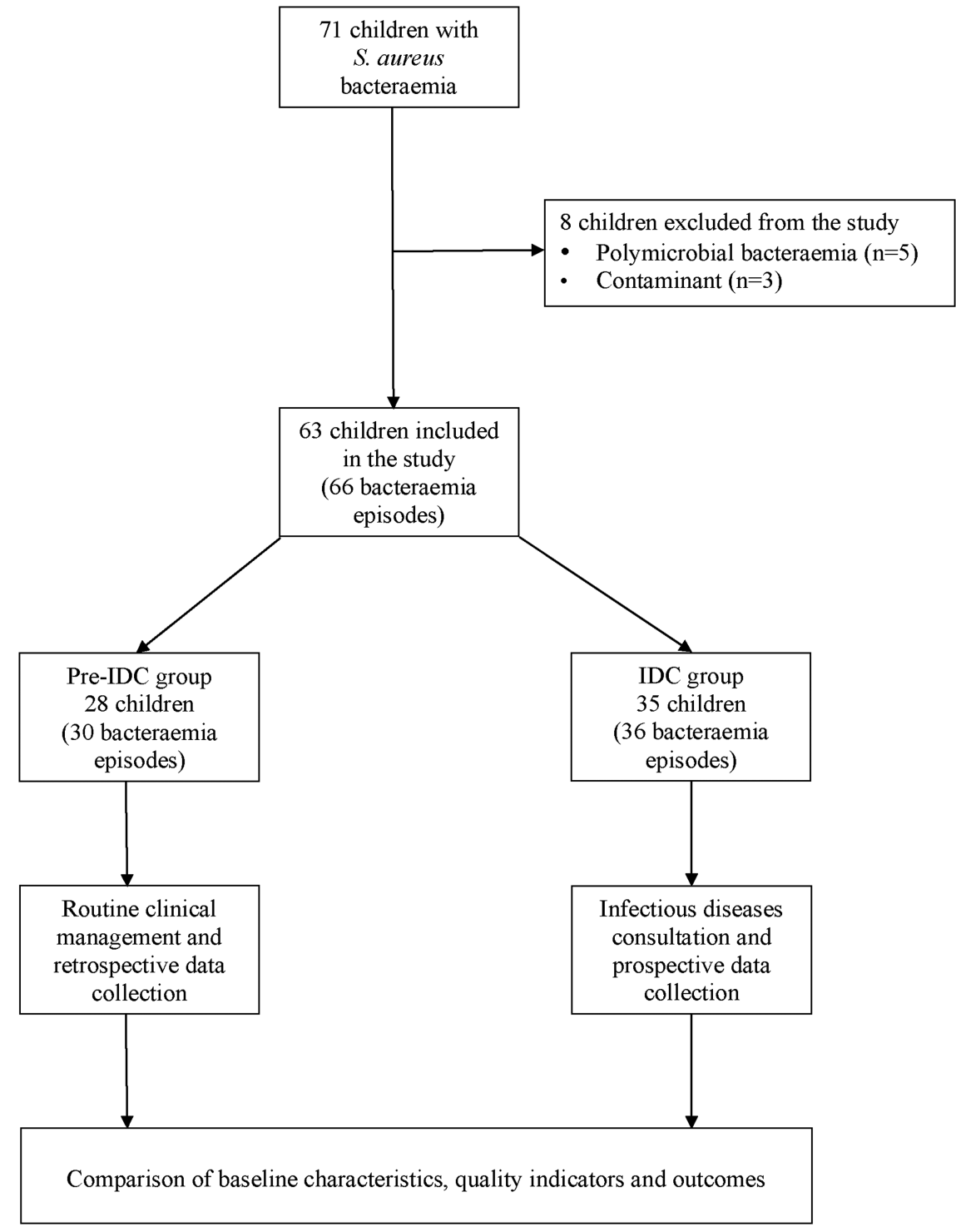

\section{Outcome of SAB}

The duration of hospital admission was similar in the pre-IDC and IDC groups, and in those with uncomplicated and complicated SAB (table 4). SAB was recorded in the discharge summary in the majority of patients in both groups. Four secondary infections were diagnosed, three in the IDC group and one in the pre-IDC group. In the IDC group, the secondary infections were tricuspid valve endocarditis (in a very low birthweight neonate with patent ductus arteriosus), pneumonia and osteomyelitis, respectively. One child in the non-IDC group developed osteomyelitis. There were three cases of recurrent bacteraemia within 90 days-two in the pre-IDC group and one in the IDC group. Three children died within 30 days of SAB, all in the IDC group, giving an overall 30-day mortality rate of $4.8 \%$. One death occurred in a child with metastatic cancer that was not attributed to SAB. The other two deaths were deemed attributable to $\mathrm{SAB}$, as blood cultures were positive at the time of death. One patient was a neonate and died prior to IDC, and the second case had cerebellar atrophy, developmental delay and was receiving total parenteral nutrition. The 90-day mortality rate was $7.9 \%$. Two patients died between 30 and 90 days post-SAB, both in the pre-IDC group. One patient had metastatic cancer, and the other had complex congenital heart disease.

\section{DISCUSSION}

To the best of our knowledge, this is the first study to systematically examine the impact of introduction of an IDC service on the management of SAB in children. We compared the clinical features, management and outcomes of all children presenting with SAB to our hospital between July 2006 and December 2012, before and after introduction of an IDC service. The main findings of the study were that patients in the IDC group were more likely to have echocardiography performed, a removable focus of infection identified and to receive a longer course of intravenous antibiotic therapy. These findings concur with those from previous studies of IDC 
Table 1 Baseline characteristics of children with Staphylococcus aureus bacteraemia included in the study

\begin{tabular}{|c|c|c|c|}
\hline Baseline variable & $\begin{array}{l}\text { Pre-IDC group } \\
\mathrm{N}=28 \text { patients (\%) }\end{array}$ & $\begin{array}{l}\text { IDC group } \\
\mathrm{N}=35 \text { patients (\%) }\end{array}$ & $\begin{array}{l}\text { Combined } \\
\mathrm{N}=63 \text { patients (\%) }\end{array}$ \\
\hline Male & $20(71.4)$ & $20(57.1)$ & $40(63.5)$ \\
\hline Female & $8(28.6)$ & 15 (42.9) & $23(36.5)$ \\
\hline Median age in years (IQR) & $4.3(0.2-9.4)$ & $3.4(0.2-12.2)$ & $3.4(0.2-10.7)$ \\
\hline Neonates & $6(20)$ & $9(25.0)$ & $15(23.8)$ \\
\hline Prematurity & $4(66.7)$ & $9(100.0)$ & $13(86.7)$ \\
\hline Median age in days (IQR) & $11(7-47)$ & $26(23-38)$ & $25(14-47)$ \\
\hline Median birth weight in grams (IQR) & $1117(630-3535)$ & $820(755-1120)$ & $830(710-1330)$ \\
\hline Congenital heart disease & $5(17.9)$ & $8(22.9)$ & $13(20.6)$ \\
\hline Chronic pulmonary disease & $3(10.7)$ & $5(14.3)$ & $8(12.7)$ \\
\hline Liver disease & 0 & $1(2.9)$ & $1(1.6)$ \\
\hline Malignancy & $7(25.0)$ & $9(25.7)$ & $16(25.4)$ \\
\hline Metastatic cancer & $2(7.1)$ & $1(2.9)$ & $3(4.8)$ \\
\hline Neurological condition & $4(14.3)$ & $13(37.1)$ & $17(27.0)$ \\
\hline Diabetes mellitus & 0 & $1(2.9)$ & $1(1.6)$ \\
\hline Skin condition & $3(10.7)$ & $1(2.9)$ & $4(6.4)$ \\
\hline Atopic dermatitis & $3(100.0)$ & & $3(75.0)$ \\
\hline Immunosuppression & $6(21.4)$ & 8 (22.9) & $14(22.2)$ \\
\hline Corticosteroid therapy & 2 & 3 & 5 \\
\hline Antineoplastic & 5 & 5 & 10 \\
\hline \multirow[t]{2}{*}{ Neutropenia } & 2 & 0 & 2 \\
\hline & $\mathrm{N}=30$ episodes (\%) & $\mathrm{N}=36$ episodes (\%) & $\mathrm{N}=66$ episodes $(\%)$ \\
\hline \multicolumn{4}{|l|}{ Mode of acquisition } \\
\hline Community-acquired & $11(36.7)$ & $10(27.8)$ & $21(31.8)$ \\
\hline Healthcare-associated & $9(30.0)$ & 7 (19.4) & $16(24.2)$ \\
\hline Hospital-acquired & $10(33.3)$ & $19(52.8)$ & 29 (43.9) \\
\hline \multicolumn{4}{|c|}{ Duration of symptoms of bacteraemia (hours) } \\
\hline $0-24$ & $21(70.0)$ & $18(50.0)$ & $39(59.1)$ \\
\hline $25-72$ & $1(3.3)$ & $6(16.7)$ & $7(10.6)$ \\
\hline$>72$ & 7 (23.3) & $10(27.8)$ & $17(25.8)$ \\
\hline Unknown & $1(3.3)$ & $2(5.6)$ & $3(4.6)$ \\
\hline \multicolumn{4}{|l|}{ Organism } \\
\hline MSSA & $28(93.3)$ & $33(91.7)$ & $61(92.4)$ \\
\hline MRSA & $2(6.7)$ & $3(8.3)$ & $5(7.6)$ \\
\hline$C$ reactive protein $(\mathrm{nmol} / \mathrm{L})$ & $333(114-890)$ & $448(181-1081)$ & $390(133-1005)$ \\
\hline White cell count $\left(10^{9} / \mathrm{L}\right)$ & $8.8(5.8-15.6)$ & $10.1(6.9-18.4)$ & $9.6(6.3-16.5)$ \\
\hline Neutrophils $\left(10^{9} / \mathrm{L}\right)$ & $5.4(3.6-9)$ & $6.3(4.9-11.8)$ & $5.7(3.6-11.8)$ \\
\hline Platelets $\left(10^{9} / \mathrm{L}\right)$ & $277(151-374)$ & $213(101-270)$ & $224(140-301)$ \\
\hline
\end{tabular}

conducted in adults with $\mathrm{SAB}$, and reflect current best practice.

Follow-up blood cultures have been recommended in adults, as prolonged bacteraemia is a predictor of complicated infection and poorer outcome in SAB. ${ }^{36}$ As a result, prolonged intravenous antibiotic therapy is recommended in patients with positive repeat blood cultures. We found that children who had a positive repeat blood culture were more likely to receive a longer course of intravenous antibiotics if they were in the IDC group.

Echocardiography was performed in a higher proportion of children in the IDC group compared with the pre-IDC group. The rates of IE in children with SAB are reported to be between $0 \%$ and $20 \%$, which is similar to rates reported in the adult population. ${ }^{1} 3681240-42$ An American study by Valente et $a t^{40}$ diagnosed IE in $20 \%$ of children with $\mathrm{SAB}(\sim 12 \%$ of whom had confirmed IE). Children with underlying congenital heart disease had a higher prevalence of confirmed or probable IE compared with those who had structurally normal hearts (53\% vs 3\%) and patients with definite IE had multiple positive blood cultures. Mortality was higher in patients with endocarditis compared with those without (40\% vs $12 \%)$. Another study from South Africa reported an IE rate of $11 \%$ in children with $\mathrm{SAB} .{ }^{12}$ Risk factors for the development of IE in children include congenital heart disease, a central IVC and persistently positive blood cultures after 24 h. $^{40}{ }^{41}$ In the UK, there are no published guidelines on the use of echocardiography in children with SAB. The Infectious Diseases Society of America guidelines for meticillin-resistant S. aureus (MRSA) bacteraemia recommend performing echocardiography in 
Table 2 Clinical features of Staphylococcus aureus bacteraemia in children included in the study

\begin{tabular}{|c|c|c|c|c|}
\hline $\begin{array}{l}\text { Focus of infection at } \\
\text { time of bacteraemia }\end{array}$ & $\begin{array}{l}\text { Pre-IDC group } \\
\mathrm{N}=30 \text { episodes (\%) }\end{array}$ & $\begin{array}{l}\text { IDC group } \\
\mathrm{N}=36 \text { episodes }(\%)\end{array}$ & $\begin{array}{l}\text { Combined } \\
\mathrm{N}=66 \text { episodes }(\%)\end{array}$ & p Value \\
\hline Unknown focus & 7 (23.3) & $2(5.6)$ & $9(13.6)$ & NS \\
\hline Intravascular catheter & $8(26.7)$ & $22(61.3)$ & $30(45.5)$ & 0.013 \\
\hline Culture confirmed & $5(62.5)$ & $11(50.0)$ & $16(53.3)$ & NS \\
\hline Thrombophlebitis & 0 & $2(5.7)$ & $2(3.0)$ & NS \\
\hline Bone/joint infection & $8(26.7)$ & $8(22.2)$ & $16(24.2)$ & NS \\
\hline Culture confirmed & $4(50.0)$ & $2(25.0)$ & $6(37.5)$ & NS \\
\hline Lung & $1(3.3)$ & $1(2.8)$ & $2(3.0)$ & NS \\
\hline Culture confirmed & 0 & $1(100.0)$ & $1(50)$ & NS \\
\hline Skin and soft tissue & 7 (23.3) & $9(25.0)$ & $16(24.2)$ & NS \\
\hline Culture confirmed & $4(57.1)$ & 8 (88.9) & $12(75.0)$ & NS \\
\hline Deep tissue abscess & $2(6.7)$ & $1(2.8)$ & $3(4.6)$ & NS \\
\hline Culture confirmed & $2(100.0)$ & 0 & $2(66.7)$ & NS \\
\hline Other focus & $2(6.7)$ & $4(11.1)$ & $6(9.1)$ & NS \\
\hline \multicolumn{5}{|l|}{ Defervescence at $72 \mathrm{~h}$} \\
\hline Yes & $18(60.0)$ & $20(55.6)$ & $38(57.6)$ & NS \\
\hline No & $11(36.7)$ & 14 (38.9) & 25 (37.9) & \\
\hline Unknown & 1 (3.3) & $2(5.6)$ & $3(4.6)$ & \\
\hline
\end{tabular}

IDC, infectious diseases consultation; NS, non-significant.

children with congenital heart disease, those with bacteraemia duration greater than 2 days or those with other clinical findings suggestive of endocarditis. ${ }^{35}$ In our study, the one child who developed tricuspid valve endocarditis was a very low birthweight premature neonate with a patent ductus arteriosus, an IVC-related infection and persistent bacteraemia. Our findings concur with these guidelines, and support the use of a risk-based strategy for the use of echocardiography in children with $\mathrm{SAB}$.

We found that a higher proportion of children had an IVC-related infection and/or a removable focus of infection in the IDC group compared with the pre-IDC group, although neither of these differences was statistically significant. It is possible that IVC was used more during the IDC period compared with the pre-IDC period. Although removable foci of infection were more frequently identified and removed in the IDC group compared with the pre-IDC group, the median time to removal was slightly longer (3 vs 2 days). In some cases, this was related to practical difficulties in removing the focus, such as re-establishing vascular access in neonates. Conversely, there were fewer patients with an unidentified focus of infection in the IDC group compared with the pre-IDC group. These findings indicate that the introduction of specialist IDC service improved the rate of diagnosis of the focus of infection in children with $\mathrm{SAB}$, suggesting that the consult service was beneficial.

The frequency of MRSA bacteraemia was only $7.6 \%$ in our study cohort. Possible explanations for this are that MRSA bacteraemia is less common in children than in adults, and that MRSA bacteraemia rates have significantly declined in the UK since 2006. In contrast, in other countries such as the USA, the incidence of MRSA bloodstream infections has been higher than in the UK, but has recently declined. In a large retrospective study

Table 3 Risk factors for Staphylococcus aureus bacteraemia in children included in the study

\begin{tabular}{|c|c|c|c|c|}
\hline Risk factor for SAB & $\begin{array}{l}\text { Pre-IDC group } \\
\mathrm{N}=30 \text { episodes (\%) }\end{array}$ & $\begin{array}{l}\text { IDC group } \\
\mathrm{N}=36 \text { episodes (\%) }\end{array}$ & $\begin{array}{l}\text { All children } \\
\mathrm{N}=66 \text { (episodes) }\end{array}$ & p Value \\
\hline Age $<1$ year & $11(36.7)$ & $14(38.9)$ & 25 (37.9) & NS \\
\hline Underlying medical condition & $16(53.3)$ & $24(66.7)$ & $40(60.6)$ & NS \\
\hline $\begin{array}{l}\text { Duration in hospital in days (IQR) } \\
\text { Prior to bacteraemia* }\end{array}$ & $11.5(7.0-21.0)$ & $19.0(12.0-37.0)$ & $16.0(8.0-24.0)$ & NS \\
\hline Prosthetic material & $14(46.7)$ & $26(72.2)$ & $40(60.6)$ & 0.04 \\
\hline Intravascular line & $13(43.3)$ & $23(63.9)$ & $36(54.6)$ & NS \\
\hline Endotracheal tube & 0 & 4 & 4 & NS \\
\hline Other & 1 & 6 & 8 & NS \\
\hline corticosteroid therapy & $2(6.7)$ & $3(8.3)$ & $5(7.6)$ & NS \\
\hline Surgery within previous 30 days & $4(13.3)$ & $4(11.1)$ & $8(12.1)$ & NS \\
\hline
\end{tabular}


Table 4 Comparison of the management and outcome of Staphylococcus aureus bacteraemia in children, preintroduction and postintroduction of an infectious disease consult service

\begin{tabular}{|c|c|c|c|}
\hline Quality indicator & $\begin{array}{l}\text { Pre-IDC group } \\
\mathrm{N}=30 \text { episodes (\%) }\end{array}$ & $\begin{array}{l}\text { IDC group } \\
\mathrm{N}=36 \text { episodes }(\%)\end{array}$ & p Value \\
\hline Median time to infectious diseases review in days (IQR) & $\begin{array}{l}\mathrm{N}=4 \\
3.5(0.5-21.5)\end{array}$ & $\begin{array}{l}\mathrm{N}=34 \\
2.0(1.0-4.0)\end{array}$ & NS \\
\hline Repeat blood culture performed & $26(86.7)$ & $32(88.9)$ & NS \\
\hline \multicolumn{4}{|l|}{ Time to repeat blood culture $(\mathrm{h})$} \\
\hline $0-48$ & 20 & 18 & NS \\
\hline $48-96$ & 8 & 13 & NS \\
\hline$>96$ & 1 & 1 & NS \\
\hline \multicolumn{4}{|l|}{ Repeat blood culture positive (h) } \\
\hline $0-48$ & 6 & 6 & NS \\
\hline $48-96$ & 3 & 4 & NS \\
\hline$>96$ & 0 & 0 & NS \\
\hline \multicolumn{4}{|l|}{ Echocardiogram performed } \\
\hline Yes & $10(33.3)$ & $29(80.6)$ & 0.0001 \\
\hline No & $20(66.7)$ & $7(19.4)$ & \\
\hline$\beta$-Lactam therapy & $27(90.0)$ & $34(94.4)$ & NS \\
\hline MSSA & 25 (92.6) & $33(97.1)$ & \\
\hline Removable focus of infection & $7(23.3)$ & 22 (43.9) & 0.003 \\
\hline Focus removed & $6(85.7)$ & $21(95.5)$ & NS \\
\hline Median time to removal in days (IQR) & $2.0(2.0-2.0)$ & $3.0(1.0-18.0)$ & NS \\
\hline Median time to appropriate antibiotics in days (IQR) & $0.0(0)$ & $0.0(0)$ & NS \\
\hline Median duration of intravenous antibiotics in days (IQR) & $13.5(7.0-21.0)$ & $18.0(15.0-29.0)$ & 0.035 \\
\hline Median duration of intravenous and/or oral antibiotics in days (IQR) & $20.5(16.0-42)$ & $19.0(15.0-29.5)$ & NS \\
\hline Complicated infection & $\begin{array}{l}\mathrm{N}=15 \text { episodes } \\
\text { Days (IQR) }\end{array}$ & $\begin{array}{l}\mathrm{N}=19 \text { episodes } \\
\text { Days (IQR) }\end{array}$ & \\
\hline Median duration of intravenous antibiotics & $14.0(6.0-21.0)$ & $22(15.0-39.0)$ & 0.02 \\
\hline Median duration of intravenous or oral antibiotics & $19.0(17.0-43.0)$ & $27.0(16.0-39.0)$ & NS \\
\hline $\begin{array}{l}\text { Median duration of intravenous antibiotics if repeat blood } \\
\text { culture positive }\end{array}$ & $13.0(6.0-14.0)$ & $19.0(15.0-27.0)$ & 0.007 \\
\hline Met standard recommendation of 28 days intravenous antibiotics (\%) & $2(13.3)$ & $8(42.1)$ & NS \\
\hline Uncomplicated infection & $\begin{array}{l}\mathrm{N}=15 \text { episodes } \\
\text { Days (IQR) }\end{array}$ & $\begin{array}{l}\mathrm{N}=17 \text { episodes } \\
\text { Days (IQR) }\end{array}$ & \\
\hline Median duration of intravenous antibiotics & $13.0(7.0-22.0)$ & $15.0(14.0-21.0)$ & NS \\
\hline Median duration of intravenous or oral antibiotics (IQR) & $22.0(14.0-32.0)$ & $18.0(14.0-29.0)$ & NS \\
\hline Met standard recommendation of 14 days (\%) & $7(46.7)$ & $13(68.4)$ & NS \\
\hline Outcomes & $\begin{array}{l}\mathrm{N}=30 \text { episodes } \\
\text { Days (IQR) }\end{array}$ & $\begin{array}{l}\mathrm{N}=36 \text { episodes } \\
\text { Days (IQR) }\end{array}$ & \\
\hline \multicolumn{4}{|l|}{ Median duration of hospital admission } \\
\hline Total & $14.0(6.0-42.0)$ & $16.5(7.5-58)$ & NS \\
\hline Complicated & $20.0(6.0-49.0)$ & $25.0(8.0-88.0)$ & NS \\
\hline Uncomplicated & $7.0(3.0-22.0)$ & $11.0(6.0-36.0)$ & NS \\
\hline SAB recorded in discharge summary & $24(80.0)$ & $29(82.9)$ & NS \\
\hline Secondary infection detected & 1 & 3 & NS \\
\hline \multicolumn{4}{|l|}{ Outcomes 30 -day post-SAB } \\
\hline Death & 0 & 3 & NS \\
\hline Recurrence & 0 & 0 & NS \\
\hline \multicolumn{4}{|l|}{ Outcomes $30-90$ days post-SAB } \\
\hline Death & 2 & 0 & NS \\
\hline Recurrence & 2 & 1 & NS \\
\hline
\end{tabular}

of over 57000 hospitalised children with $S$. aureus infections, $51 \%$ had MRSA and $61 \%$ had MRSA skin and soft tissue infections. The incidence of skin and soft tissue infections, pneumonia, osteomyelitis and bacteraemia increased over time but overall mortality was low $(1 \%)$. Thus, the findings of our study may not be generalisable to other settings where the epidemiology and outcomes of MRSA bacteraemia are different. 
In terms of duration of antimicrobial therapy, we found that patients in the IDC group received longer courses of intravenous antibiotics in complicated infection, compared with patients in the pre-IDC group. This concurs with findings from studies in adults with $\mathrm{SAB}$, and suggests that specialist infectious diseases review may be beneficial in ensuring that clinical management recommendations, such as the length of intravenous antimicrobial therapy, are applied. There were, however, no differences in mortality observed between the pre-IDC and IDC groups. The most likely explanation for this was the small study population, combined with a low mortality rate, which meant that a large number of patients would be required to demonstrate even a small difference in mortality. Furthermore, the overall duration of antibiotic therapy was similar in the two groups. It may be that total duration of antibiotic therapy is more important than the route of administration, provided that adequate concentrations are achieved in the bloodstream. Indeed, studies to examine this very question, comparing short versus long courses of intravenous antibiotics in SAB in adults, are ongoing. Finally, although a removable focus of infection was identified more frequently in the IDC group, the likelihood of removal and the time to removal did not differ; this may also explain the lack of difference in outcome between the two groups.

We acknowledge several limitations to our study. The study population was small and the differences in diagnosis and management that we observed in the two groups did not translate into differences in outcome, for reasons discussed above. The retrospective data collection during the pre-IDC period (2006-2009) carries a risk of incomplete recording of data and potential bias. There were, however, no differences in baseline characteristics between the two groups in terms of age, gender, underlying comorbidities or focus of infection. The only exception was a higher frequency of IVC-related infections in the IDC group, which may have been under-recorded in the pre-IDC period and/or diagnosed more frequently in the IDC period.

In conclusion, we found that introduction of IDC for paediatric SAB resulted in improvements in management and a more consistent approach to care across the paediatric service. These findings concur with those of previous studies of IDC in adults with SAB. Our findings support the use of a targeted approach to echocardiography in SAB in children, particularly for patients with risk factors for complicated disease. Despite improvements in the investigation and clinical management, we did not find any differences in the development of secondary infections, recurrent bacteraemia or death between the two groups. The most likely explanation for this is the small study population and larger prospective studies are required to validate our findings and to determine the optimal strategies for investigation and management of paediatric SAB.
Author affiliations

${ }^{1}$ Department of Medicine, University of Cambridge, Cambridge, UK

${ }^{2}$ Department of Microbiology, Cambridge University Hospitals NHS Foundation Trust, Cambridge, UK

${ }^{3}$ Public Health England, Clinical Microbiology and Public Health Laboratory, Cambridge, UK

${ }^{4}$ Department of Infectious Diseases, Cambridge University Hospitals NHS

Foundation Trust, Cambridge, UK

${ }^{5}$ Department of Paediatrics, Cambridge University Hospitals NHS Foundation

Trust, Cambridge, UK

${ }^{6}$ Mahidol Oxford Research Unit, Mahidol University, Bangkok, Thailand

${ }^{7}$ Wellcome Trust Sanger Institute, Hinxton, UK

Contributors RBS, TG, EJN, SHA and DRO were part of the infectious diseases consultation service; they collected the data and contributed to the writing of the manuscript. WK was part of the clinical care team and contributed to the writing of the manuscript. SJP and MET conceived and supervised the study and contributed to the writing of the manuscript. All authors approved the final manuscript.

Funding This work was supported by grants from the UK Clinical Research Collaboration (UKCRC) Translational Infection Research Initiative (TIRI); the Medical Research Council (G1000803), with contributions from the Biotechnology and Biological Sciences Research Council, the National Institute for Health Research (NIHR) on behalf of the UK Department of Health, and the Chief Scientist of the Scottish Government Health Directorate; the Public Health England; and the NIHR Cambridge Biomedical Research Centre. MET is a Clinician Scientist Fellow funded by the Academy of Medical Sciences at the Health Foundation.

Competing interests None.

Ethics approval The study protocol received approval from the University of Cambridge Human Biology Research Ethics Committee, and from the CUH Research and Development Department.

Provenance and peer review Not commissioned; externally peer reviewed.

Data sharing statement No additional data are available.

Open Access This is an Open Access article distributed in accordance with the terms of the Creative Commons Attribution (CC BY 3.0) license, which permits others to distribute, remix, adapt and build upon this work, for commercial use, provided the original work is properly cited. See: http:// creativecommons.org/licenses/by/3.0/

\section{REFERENCES}

1. Burke RE, Halpern MS, Baron EJ, et al. Pediatric and neonatal Staphylococcus aureus bacteremia: epidemiology, risk factors, and outcome. Infect Control Hosp Epidemiol 2009;30:636-44.

2. Mylotte JM, Tayara A. Staphylococcus aureus bacteremia: predictors of 30-day mortality in a large cohort. Clin Infect Dis 2000;31:1170-4.

3. Healy CM, Palazzi DL, Edwards MS, et al. Features of invasive staphylococcal disease in neonates. Pediatrics 2004;114:953-61.

4. Frederiksen MS, Espersen F, Frimodt-Møller N, et al. Changing epidemiology of pediatric Staphylococcus aureus bacteremia in Denmark from 1971 through 2000. Pediatr Infect Dis J 2007;26:398-405.

5. Hakim H, Mylotte JM, Faden $\mathrm{H}$. Morbidity and mortality of Staphylococcal bacteremia in children. Am J Infect Control 2007;35:102-5.

6. Denniston S, Riordan FAI. Staphylococcus aureus bacteraemia in children and neonates: a 10 year retrospective review. $J$ Infect 2006;53:387-93.

7. Hill PC, Wong CGS, Voss LM, et al. Prospective study of 125 cases of Staphylococcus aureus bacteraemia in children in New Zealand. Pediatr Infect Dis J 2001;20:868-73.

8. Gray J, O'Donoghue B. Bacteraemia with meticillin-susceptible Staphylococcus aureus in an English children's hospital. J Hosp Infect 2011;78:158-9.

9. Groome MJ, Albrich WC, Wadula J, et al. Community-onset Staphylococcus aureus bacteraemia in hospitalised African children: high incidence in HIV-infected children and high prevalence of multidrug resistance. Paediatr Int Child Health 2012;32:140-6. 
10. Suryati BA, Watson M. Staphylococcus aureus bacteraemia in children: a 5-year retrospective review. J Paediatr Child Health 2002;38:290-4.

11. Wyllie DH, Crook DW, Peto TEA. Mortality after Staphylococcus aureus bacteraemia in two hospitals in Oxfordshire, 1997-2003: cohort study. BMJ 2006;333:281.

12. Friendland IR, du Plessis J, Cilliers A. Cardiac complications in children with Staphylococcus aureus bacteremia. J Pediatr 1995;127:476-8.

13. Carrillo-Marquez MA, Hulten KG, Mason EO, et al. Clinical and molecular epidemiology of Staphylococcus aureus catheter-related bacteremia in children. Pediatr Infect Dis J 2010;29:410-14.

14. Fowler VG, Sanders LL, Sexton DJ, et al. Outcome of Staphylococcus aureus bacteremia according to compliance with recommendations of infectious diseases specialists: experience with 244 patients. Clin Infect Dis 1998;27:478-86.

15. Lillie $\mathrm{P}$, Moss $\mathrm{P}$, Thaker $\mathrm{H}$, et al. Development, impact and outcomes of the Hull Bacteraemia Service. QJM 2008;101:889-98.

16. Nagao M, linuma $Y$, Saito $T$, et al. Close cooperation between infectious disease physicians and attending physicians can result in better management and outcome for patients with Staphylococcus aureus bacteraemia. Clin Microbiol Infect 2010;16:1783-8.

17. Robinson JO, Pozzi-Langhi S, Phillips $\mathrm{M}$, et al. Formal infectious diseases consultation is associated with decreased mortality in Staphylococcus aureus bacteraemia. Eur J Clin Microbiol Infect Dis 2012;31:2421-8.

18. Jenkins TC, Price CS, Sabel AL, et al. Impact of routine infectious diseases service consultation on the evaluation, management, and outcomes of Staphylococcus aureus bacteremia. Clin Infect Dis 2008;46:1000-8.

19. Choi S-H, Cho SY, Park J-H, et al. Impact of infectious-disease specialist consultations on outcomes of Staphylococcus aureus bacteremia in a hospital with a low volume of patients with $\mathrm{S}$. aureus bacteremia. J Infect 2011;62:181-5

20. Kaech C, Elzi L, Sendi P, et al. Course and outcome of Staphylococcus aureus bacteraemia: a retrospective analysis of 308 episodes in a Swiss tertiary-care centre. Clin Microbiol Infect 2006;12:345-52.

21. Honda $\mathrm{H}$, Krauss MJ, Jones JC, et al. The value of infectious diseases consultation in Staphylococcus aureus bacteremia. Am J Med 2010;123:631-7.

22. Rieg S, Peyerl-Hoffmann G, de With K, et al. Mortality of S. aureus bacteremia and infectious diseases specialist consultation-a study of 521 patients in Germany. J Infect 2009;59:232-9.

23. De La Blanchardière $A$, Boutemy J, Thibon $P$, et al. Clinical benefit of infectious diseases consultation: a monocentric prospective cohort study. Infection 2012;40:501-7.

24. Forsblom E, Ruotsalainen E, Ollgren J, et al. Telephone consultation cannot replace bedside infectious disease consultation in the management of Staphylococcus aureus bacteremia. Clin Infect Dis 2013;56:527-35

25. Tissot F, Calandra T, Prod'hom G, et al. Impact of infectious diseases consultation on outcome of methicillin-resistant Staphylococcus aureus bacteraemia in a tertiar centre: a ten-year experience. Clin Microbiol Infect 2011;17:S420-1.
26. Minton J, Clayton J, Sandoe J, et al. Improving early management of bloodstream infection: a quality improvement project. $B M J$ 2008;336:440-3.

27. Lundberg J, Nettleman MD, Costigan M, et al. Staphylococcus aureus bacteraemia: the cost effectiveness of long-term therapy associated with infectious diseases consultation. Clin Perform Qual Health Care 1998;6:9-11.

28. Enoch DA, Cooke FJ, Guha S, et al. Thermostable nuclease: a study of clinical impact. J Antimicrob Chemother 2008;61:754-5.

29. BSAC. BSAC Methods for Antimicrobial Susceptibility Testing, Version 12 May 2013. http://bsac.org.uk/wp-content/uploads/2012/ 02/Version-12-Apr-2013 final.pdf

30. Libman H, Arbeit R. Complications associated with Staphylococcus aureus bacteraemia. Arch Intern Med 1984;144:541-5.

31. Horan TC, Andrus M, Dudeck MA. CDC/NHSN surveillance definition of healthcare-associated infection and criteria for specific types of infections in the acute care setting. 2008;36:309-32.

32. Li JS, Sexton DJ, Mick N, et al. Proposed modifications to the Duke criteria for the diagnosis of infective endocarditis. Clin Infect Dis 2000;30:633-8.

33. Durack DT, Lukes AS, Bright DK. New criteria for diagnosis of infective endocarditis: utilization of specific echocardiographic findings. Am J Med 1994;96:200-9.

34. Friedman ND, Kaye KS, Stout JE, et al. Health care-associated bloodstream infections in adults: a reason to change the accepted definition of community-acquired infections. Ann Intern Med 2002;10:791-7.

35. Liu C, Bayer A, Cosgrove SE, et al. Clinical practice guidelines by the infectious diseases society of America for the treatment of methicillin-resistant Staphylococcus aureus infections in adults and children. Clin Infect Dis 2011;52:e18-55.

36. Fowler VG, Olsen MK, Corey GR, et al. Clinical identifiers of complicated Staphylococcus aureus bacteraemia. Arch Inter Med 2003;163:2066-72.

37. Mermel LA, Allon M, Bouza E, et al. Clinical practice guidelines for the diagnosis and management of intravascular catheter-related infection: 2009 Update by the Infectious Diseases Society of America. Clin Infect Dis 2009;49:1-45.

38. Thwaites GE, Edgeworth JD, Gkrania-Klotsas E, et al. Clinical management of Staphylococcus aureus bacteraemia. Lancet Infect Dis 2011;11:208-22.

39. Calain $\mathrm{P}$, Krause $\mathrm{KH}$, Vaudaux $\mathrm{P}$, et al. Early termination of a prospective, randomized trial comparing teicoplanin and flucloxacillin for treating severe staphylococcal infections. J Infect Dis 1987;155:187-91.

40. Valente AM, Jain R, Scheurer M, et al. Frequency of infective endocarditis among infants and children with Staphylococcus aureus bacteremia. Pediatrics 2005;115:e15-19.

41. Ross $A C$, Toltzis $P$, O'Riordan MA, et al. Frequency and risk factors for deep focus of infection in children with Staphylococcus aureus bacteremia. Pediatr Infect Dis J 2008;27:396-9.

42. Johnson AP, Sharland $M$, Goodall $C M$, et al. Enhanced surveillance of methicillin-resistant Staphylococcus aureus (MRSA) bacteraemia in children in the UK and Ireland. Arch Dis Child 2010;95:781-5. 